\title{
Monotone iterations for differential problems
}

\author{
Tadeusz Jankowski
}


Mathematical Notes, Miskolc, Vol. 2., No. 1., (2001), pp. 31-38

\title{
MONOTONE ITERATIONS FOR DIFFERENTIAL PROBLEMS
}

\author{
TADEUSZ JANKOWSKI \\ Department of Differential Equations, Technical University of Gdansk \\ 11/12 Narutowicz Str., 80-952 Gdansk, Poland \\ tjank@mif.pg.gda.pl
}

[Received March 20, 2001]

\begin{abstract}
The purpose of this survey paper is to formulate existence results for parameterized non-linear boundary value problems employing the method of upper and lower solutions and the method of quasilinearization.
\end{abstract}

Mathematical Subject Classification: 34B15

Keywords: Non-linear parametrized boundary value problem, upper and lower solutions, quasilinearization

\section{Introduction}

Let us consider the differential equation of first order with a parameter of the form

$$
\left\{\begin{array}{c}
x^{\prime}(t)=f(t, x(t), \lambda), \quad t \in J=[0, b] \\
x(0)=k_{0} \in R \quad G(x(b), \lambda)=0
\end{array}\right.
$$

where $f \in C(J \times R \times R, R)$, and $G \in C(R \times R, R)$. By a solution of (1.1) we mean a pair $(x, \lambda) \in C^{1}(J, R) \times R$ for which problem (1.1) is satisfied. Problems with a parameter have been considered for many years. Some of them appeared as mathematical models of physical systems (see, for example [16]).

An important area of research in the qualitative theory of differential equations is the study of existence of solutions. Existence/uniqueness theorems for problem (1.1) can be formulated under the assumption that $f$ and $G$ satisfy the Lipschitz condition with respect to the last two variables with suitable Lipschitz constants or Lipschitz functions (see $[1,2,8,15])$. In the above mentioned papers the method of successive approximations and comparison technique are used to obtain sufficient conditions on existence/uniqueness solutions for problems with a parameter. An interesting technique for proving existence results is the method based on upper and lower solutions (for details, see [9]). The purpose of this paper is to formulate existence results for problem (1.1) employing the method of upper and lower solutions. As we see one-sided Lipschitz conditions are imposed on $f$ and $G$ (Theorems 1 and 2). If we apply the method of quasilinearization (for details, see [10]), then we can construct monotone sequences which converge quadratically to the unique solution of problem (1.1). We show that if we replace $f$ and $G$ by the sum of two corresponding functions, 
then monotone iterations converge quadratically to the unique solution of problem (3.1) (see Theorem 3). Theorem 4 generalizes this result. Theorem 5 concerns a more general case when some Lipschiz functions are added to the right-hand sides of problem (3.1). In this case the convergence is only semi-quadratic. This is a survey paper connected with the above technique for problems of type (1.1).

\section{Extremal solutions of problem (1.1)}

A pair $(v, \alpha) \in C^{1}(J, R) \times R$ is said to be a lower solution of (1.1) if

$$
\left\{\begin{array}{c}
v^{\prime}(t) \leq f(t, v(t), \alpha), \quad t \in J \\
v(0)=k_{0} \quad 0 \leq G(v(b), \alpha)
\end{array}\right.
$$

and an upper solution of (1.1) if the above inequalities are reversed.

The next two theorems give constructive sufficient conditions when problem (1.1) has minimal and maximal solutions. Note that one-sided Lipschitz conditions are imposed on $f$ and $G$.

Theorem 1 (see [4]). Assume that $f \in C(J \times R \times R, R), G \in C(R \times R, R)$ and

$1^{o}\left(y_{0}, \lambda_{0}\right),\left(z_{0}, \gamma_{0}\right) \in C^{1}(J, R) \times R$ are lower and upper solutions of problem (1.1) such that $y_{0}(t) \leq z_{0}(t), \quad t \in J$ and $\lambda_{0} \leq \gamma_{0}$,

$2^{o} f$ is nondecreasing with respect to the last variable,

$2^{o} f(t, \bar{u}, \lambda)-f(t, u, \lambda) \geq-M(\bar{u}-u)=$ for $y_{0} \leq u \leq \bar{u} \leq z_{0}$ with $M \geq 0$,

$4^{o} G(\bar{u}, \lambda)-G(u, \lambda) \geq Q(\bar{u}-u)$ for $y_{0} \leq u \leq \bar{u} \leq z_{0}, \quad \lambda_{0} \leq \lambda \leq \gamma_{0}$ with $Q \geq 0$,

$5^{o} G(u, \bar{\lambda})-G(u, \lambda) \geq=-N(\bar{\lambda}-\lambda)$ for $\lambda_{0} \leq \lambda \leq \bar{\lambda} \leq \gamma_{0}, \quad y_{0} \leq u \leq z_{0}$ with $N>0$.

Then there exist monotone sequences $\left\{y_{n}, \lambda_{n}\right\},\left\{z_{n}, \gamma_{n}\right\}$ such that $y_{n}(t) \rightarrow y(t), z_{n}(t)$ $\rightarrow z(t), \quad t \in J$ and $\lambda_{n} \rightarrow \lambda, \quad \gamma_{n} \rightarrow \gamma$ as $n \rightarrow \infty$ and this convergence is uniform and monotone on $J$. Moreover, $(y, \lambda)$ and $(z, \gamma)$ are minimal and maximal solutions of problem (1.1), respectively.

Remark 1. We observe that the special cases when $f$ is monotone nondecreasing with respect to the second variable and $G$ is monotone nondecreasing with respect to the first and second variables are covered by Theorem 1 . To see this, it is enough to put $M=0$ in condition $3^{\circ}$, and $Q=0$ in condition $4^{\circ}$. If $G$ is monotone nondecreasing with respect to the second variable, then there exists $N>0$ such that for $\bar{\lambda} \geq \lambda$ we have

$$
G(u, \bar{\lambda})-G(u, \lambda) \geq 0 \geq-N(\bar{\lambda}-\lambda),
$$

which proves that condition $5^{\circ}$ holds also.

Example (see [4]). Let $J=[0,1]$,

$$
\left\{\begin{array}{c}
x^{\prime}(t)=x^{2}(t)+\frac{1}{3}\left[\sin ^{2} x(t)+t^{2}+g(\lambda)\right] \equiv f(t, x(t), \lambda), \quad t \in J, x(0)=0 \\
0=x(1.1)+\frac{1}{10} \sin [x(1.1)+\lambda]-\lambda \equiv G(x(1.1), \lambda)
\end{array}\right.
$$


where

$$
g(\lambda)=\left\{\begin{array}{ccc}
0 & \text { if } & \lambda \leq 0 \\
\lambda^{2} & \text { if } & 0<\lambda<1 \\
1 & \text { if } & 1 \leq \lambda
\end{array}\right.
$$

Since

$$
\frac{1}{3} t^{2} \leq f(t, x, \lambda) \leq 1+x^{2}
$$

then

$$
y_{0}(t)=\frac{1}{9} t^{3}, \quad z_{0}(t)=\tan t, \quad t \in J
$$

Similarly,

$$
\frac{1}{9}-\frac{1}{10}-\lambda \leq x(1.1)-\frac{1}{10}-\lambda \leq G(x(1.1), \lambda) \leq x(1.1)+\frac{1}{10}-\lambda \leq \tan 1+\frac{1}{10}-\lambda,
$$

then

$$
\lambda_{0}=\frac{1}{90} \approx 0.01, \quad \gamma_{0}=\tan 1+\frac{1}{10} \approx 1.66 .
$$

Note that $\left(y_{0}, \lambda_{0}\right),\left(z_{0}, \gamma_{0}\right)$ are lower and upper solutions of our problem, respectively, and $y_{0}(t) \leq z_{0}(t), t \in J, \quad \lambda_{0}<\gamma_{0}$. Condition $1^{\circ}$ of Theorem 1 holds since $g$ is nondecreasing in $\lambda$. Let $\bar{u} \geq u$ and $\bar{\lambda} \geq \lambda$. Using the mean value theorem we see that

$$
\begin{gathered}
f(t, \bar{u}, \lambda)-f(t, u, \lambda)=(\bar{u})^{2}-u^{2}+\frac{1}{3}\left[\sin ^{2} \bar{u}-\sin ^{2} u\right] \geq \frac{1}{3}\left[\sin ^{2} \bar{u}-\sin ^{2} u\right] \geq-\frac{1}{3}(\bar{u}-u), \\
G(\bar{u}, \lambda)-G(u, \lambda)=\bar{u}-u+\frac{1}{10}[\sin (\bar{u}+\lambda)-\sin (u+\lambda)] \geq \frac{9}{10}(\bar{u}-u), \\
G(u, \bar{\lambda})-G(u, \lambda)=\frac{1}{10}[\sin (u+\bar{\lambda})-\sin (u+\lambda)]-(\bar{\lambda}-\lambda) \geq-\frac{11}{9}(\bar{\lambda}-\lambda) .
\end{gathered}
$$

All assumptions of Theorem 1 hold, its assertion is satisfied for this problem.

Theorem 2 (see [4]). Let $f \in C(J \times R \times R, R), G \in C(R \times R, R)$ and $\}$

$1^{o}\left(y_{0}, \lambda_{0}\right),\left(z_{0}, \gamma_{0}\right) \in C^{1}(J, R) \times R$ are lower and upper solutions of problem (1.1) such that $y_{0}(t) \leq z_{0}(t), \quad t \in J$ and $\lambda_{0} \leq \gamma_{0}$,

$2^{o} f(t, u, \bar{\lambda})-f(t, u, \lambda) \geq=Q(\bar{\lambda}-\lambda)$ for $\lambda_{0} \leq \lambda \leq \bar{\lambda} \leq \gamma_{0}, y_{0} \leq u \leq z_{0}$ with $Q \geq 0$,

$3^{o} f(t, \bar{u}, \lambda)-f(t, u, \lambda) \geq-M(\bar{u}-u)=$ for $y_{0} \leq u \leq \bar{u} \leq z_{0}$ with $M \geq 0$,

$4^{o} G$ is nondecreasing with respect to the first variable,

$5^{o} G(u, \bar{\lambda})-G(u, \lambda) \geq=-N(\bar{\lambda}-\lambda)$ for $\lambda_{0} \leq \lambda \leq \bar{\lambda} \leq \gamma_{0}, y_{0} \leq u \leq z_{0}$, with $N>0$.

Then there exist monotone sequences $\left\{y_{n}, \lambda_{n}\right\},\left\{z_{n}, \gamma_{n}\right\}$ such that $y_{n}(t) \rightarrow y(t)$, $z_{n}(t) \rightarrow z(t), \quad t \in J$ and $\lambda_{n} \rightarrow \lambda, \quad \gamma_{n} \rightarrow \gamma$ as $n \rightarrow \infty$ and this convergence is uniform and monotone on $J$. Moreover, $(y, \lambda)$ and $(z, \gamma)$ are minimal and maximal solutions of problem (1.1), respectively.

Remark 2. Our results may be extended to a finite system of differential equations of type (1.1). Although this extension follows similar ideas, such a case requires special 
care since we need to split variables as it is evident in the technique of monotone iterations.

\section{Quasilinearization method}

Let $y_{0}, z_{0} \in C^{1}(J, R)$ and $\lambda_{0}, \gamma_{0} \in R$ such that $y_{0}(t) \leq z_{0}(t)$ on $J$ and $\lambda_{0} \leq \gamma_{0}$. Define the closed sets:

$$
\begin{gathered}
\Omega=\left\{(t, y, \lambda): t \in J, \quad y_{0}(t) \leq y \leq z_{0}(t), \quad \lambda_{0} \leq \lambda \leq \gamma_{0}\right\} \\
\bar{\Omega}=\left\{(y, \lambda): y_{0}(b) \leq y \leq z_{0}(b), \quad \lambda_{0} \leq \lambda \leq \gamma_{0}\right\}
\end{gathered}
$$

Now, instead of (1.1), we shall consider the following problem

$$
\left\{\begin{array}{c}
x^{\prime}(t)=f(t, x(t), \lambda)+g(t, x(t), \lambda), \quad t \in J, \quad x(0)=k_{0} \in R \\
G(x(b), \lambda)+H(x(b), \lambda)=0,
\end{array}\right.
$$

where $f, g \in C(J \times R \times R, R), G, H \in C(R \times R, R)$.

The method of quasilinearization offers monotone sequences of approximate solutions that converge quadratically to the unique solution. This problem is considered in the next theorems using less restrictive assumptions.

Theorem 3 (see [5]). Let $f, g, f_{y}, g_{y}, f_{\lambda}, g_{\lambda} \in C(\Omega, R), G, H, G_{y}, H_{y}, G_{\lambda}, H_{\lambda} \in$ $C(\bar{\Omega}, R)$. Assume that:

$1^{o}\left(y_{0}, \lambda_{0}\right),\left(z_{0}, \gamma_{0}\right) \in C^{1}(J, R) \times R$ are lower and upper solutions of problem (3.1), respectively, and such that $y_{0}(t) \leq z_{0}(t), t \in J$ and $\lambda_{0} \leq \gamma_{0}$,

$2^{o}-G_{\lambda}(u, v) \geq K_{1}, \quad-H_{\lambda}(u, v) \geq K_{2}, K=K_{1}+K_{2}>0, \bar{L}_{1} \leq G_{y}(u, v) \leq L_{1}, \quad \bar{L}_{2} \leq$ $H_{y}(u, v) \leq L_{2}, \bar{L}=\bar{L}_{1}+\bar{L}_{2} \geq 0, \bar{M}_{1} \leq f_{\lambda}(t, \bar{u}, \bar{v}) \leq M_{1}, \quad \bar{M}_{2} \leq g_{\lambda}(t, \bar{u}, \bar{v}) \leq M_{2}, \bar{M}=$ $\bar{M}_{1}+\bar{M}_{2} \geq 0$

for $(u, v) \in \bar{\Omega}, \quad(t, \bar{u}, \bar{v}) \in \Omega$, and put $L=L_{1}+L_{2}, \quad M=M_{1}+M_{2}$,

$3^{o}\left|f_{y}(t, u, v)\right| \leq N_{1}, \quad\left|g_{y}(t, u, v)\right| \leq N_{2}=$ for $(t, u, v) \in \Omega$, and put $N=N_{1}+N_{2}$,

$4^{o} \quad S(b)<1$, where

$$
S(t)=\left\{\begin{array}{cc}
\frac{M L}{K} t & \text { if } \quad N=0 \\
\frac{M L}{K N}[\exp (N t)-1] & \text { if } \quad N>0
\end{array}\right.
$$

$5^{o} f_{y y}, f_{y \lambda}, f_{\lambda y}, f_{\lambda \lambda}, g_{y y}, g_{y \lambda}, g_{\lambda y}, g_{\lambda \lambda}$ exist, are continuous and satisfy the relations:

$$
\begin{aligned}
& f_{y y}(t, u, v) \geq 0, \quad f_{y \lambda}(t, u, v) \geq 0, \quad f_{\lambda \lambda}(t, u, v) \geq 0 \text { for }(t, u, v) \in \Omega, \\
& g_{y y}(t, u, v) \leq 0, \quad g_{y \lambda}(t, u, v) \leq 0, \quad g_{\lambda \lambda}(t, u, v) \leq 0 \text { for }(t, u, v) \in \Omega,
\end{aligned}
$$

$6^{o} G_{y y}, G_{y \lambda}, G_{\lambda y}, G_{\lambda \lambda}, H_{y y}, H_{\lambda y}, H_{y \lambda}, H_{\lambda \lambda}$ exist, are continuous and satisfy the relations:

$$
G_{y y}(u, v) \geq 0, G_{y \lambda}(u, v) \geq 0, G_{\lambda \lambda}(u, v) \geq 0 \text { for }(u, v) \in \bar{\Omega},
$$




$$
H_{y y}(u, v) \leq 0, \quad H_{y \lambda}(u, v) \leq 0, \quad H_{\lambda \lambda}(u, v) \leq 0 \text { for }(u, v) \in \bar{\Omega} .
$$

Then there exist monotone sequences $\left\{y_{n}\right\},\left\{z_{n}\right\},\left\{\lambda_{n}\right\},\left\{\gamma_{n}\right\}$ which converge uniformly to the unique solution $(x, \lambda)$ of problem $(3.1)$ and the convergence is quadratic, i.e.,

$$
\begin{aligned}
& \max _{t \in J}\left|x(t)-y_{n+1}(t)\right| \leq a_{1} \max _{t \in J}\left|x(t)-y_{n}(t)\right|^{2}+a_{2} \max _{t \in J}\left|x(t)-z_{n}(t)\right|^{2} \\
&+a_{3}\left|\lambda-\lambda_{n}\right|^{2}+a_{4}\left|\lambda-\gamma_{n}\right|^{2}, \\
&\left|\lambda-\lambda_{n+1}\right| \leq b_{1} \max _{t \in J}\left|x(t)-y_{n}(t)\right|^{2}+b_{2} \max _{t \in J}\left|x(t)-z_{n}(t)\right|^{2} \\
&+b_{3}\left|\lambda-\lambda_{n}\right|^{2}+b_{4}\left|\lambda-\gamma_{n}\right|^{2}, \\
& \max _{t \in J}\left|x(t)-z_{n+1}(t)\right| \leq \bar{a}_{1} \max _{t \in J}\left|x(t)-y_{n}(t)\right|^{2}+\bar{a}_{2} \max _{t \in J}\left|x(t)-z_{n}(t)\right|^{2} \\
&+\bar{a}_{3}\left|\lambda-\lambda_{n}\right|^{2}+\bar{a}_{4}\left|\lambda-\gamma_{n}\right|^{2}, \\
&\left|\lambda-\gamma_{n+1}\right| \leq \bar{b}_{1} \max _{t \in J}\left|x(t)-y_{n}(t)\right|^{2}+\bar{b}_{2} \max _{t \in J}\left|x(t)-z_{n}(t)\right|^{2} \\
&+\bar{b}_{3}\left|\lambda-\lambda_{n}\right|^{2}+\bar{b}_{4}\left|\lambda-\gamma_{n}\right|^{2}
\end{aligned}
$$

for some nonnegative constants $a_{i}, b_{i}, \bar{a}_{i}, \bar{b}_{i}, i=1,2,3,4$.

\section{Generalization of the quasilinearization method}

We will use the following notation $f \in C^{0,2,2}(\Omega, R)$ which means that $f, f_{y}, f_{\lambda}, f_{y y}$, $f_{y \lambda}, f_{\lambda y}, f_{\lambda \lambda} \in C(\Omega, R)$. The next theorem generalizes the result of Theorem 3 .

Theorem 4 (see $[6]$ ). Let $f, g \in C^{0,2,2}(\Omega, R), G, H \in C^{2}(\bar{\Omega}, R)$. Assume that:

$1^{o}\left(y_{0}, \lambda_{0}\right),\left(z_{0}, \gamma_{0}\right) \in C^{1}(J, R) \times R$ are lower and upper solutions of problem (3.1), respectively, and such that $y_{0}(t) \leq z_{0}(t), t \in J$ and $\lambda_{0} \leq \gamma_{0}$,

$2^{o}-G_{\lambda}(u, v) \geq K_{1}, \quad-H_{\lambda}(u, v) \geq K_{2}$ and $K=K_{1}+K_{2}>0$,

$\bar{L}_{1} \leq G_{y}(u, v) \leq L_{1}, \quad \bar{L}_{2} \leq H_{y}(u, v) \leq L_{2}, \bar{L}=\bar{L}_{1}+\bar{L}_{2}$,

$\bar{M}_{1} \leq f_{\lambda}(t, \bar{u}, \bar{v}) \leq M_{1}, \quad \bar{M}_{2} \leq g_{\lambda}(t, \bar{u}, \bar{v}) \leq M_{2}, \bar{M}=\bar{M}_{1}+\bar{M}_{2}$ for $(u, v) \in$ $\bar{\Omega}, \quad(t, \bar{u}, \bar{v}) \in \Omega$, and put $L=L_{1}+L_{2}, \quad M=M_{1}+M_{2}$,

$3^{o}\left|f_{y}(t, u, v)\right| \leq N_{1}, \quad\left|g_{y}(t, u, v)\right| \leq N_{2}$ for $(t, u, v) \in \Omega$, and put $N=N_{1}+N_{2}$,

$4^{o} \quad S(b)<1$, where $S$ is defined as in condition $4^{o}$ of Theorem 3 ,

$5^{o} \quad \Psi, \Phi \in C^{0,2,2}(\Omega, R)$ and satisfy the relations for $(t, u, v) \in \Omega$ with $F=f+\Phi,=$ $T=g+\Psi$

$$
\begin{gathered}
\Phi_{y y}(t, u, v) \geq 0, \quad \Phi_{y \lambda}(t, u, v) \geq 0, \quad \Phi_{\lambda \lambda}(t, u, v) \geq 0, \\
F_{y y}(t, u, v) \geq 0, \quad F_{y \lambda}(t, u, v) \geq 0, \quad F_{\lambda \lambda}(t, u, v) \geq 0, \\
\Psi_{y y}(t, u, v) \leq 0, \quad \Psi_{y \lambda}(t, u, v) \leq 0, \quad \Psi_{\lambda \lambda}(t, u, v) \leq 0,
\end{gathered}
$$




$$
T_{y y}(t, u, v) \leq 0, \quad T_{y \lambda}(t, u, v) \leq 0, \quad T_{\lambda \lambda}(t, u, v) \leq 0
$$

$6^{o} \Delta, \Gamma \in C^{2}(\bar{\Omega}, R)$ and satisfy the relations for $(u, v) \in \bar{\Omega}$ with $P=G+\Delta,=Q=$ $H+\Gamma$

$$
\begin{gathered}
\Delta_{y y}(u, v) \geq 0, \quad \Delta_{y \lambda}(u, v) \geq 0, \quad \Delta_{\lambda \lambda}(u, v) \geq 0, \\
P_{y y}(u, v) \geq 0, \quad P_{y \lambda}(u, v) \geq 0, \quad P_{\lambda \lambda}(u, v) \geq 0, \\
\Gamma_{y y}(u, v) \leq 0, \quad \Gamma_{y \lambda}(u, v) \leq 0, \quad \Gamma_{\lambda \lambda}(u, v) \leq 0, \\
Q_{y y}(u, v) \leq 0, \quad Q_{y \lambda}(u, v) \leq 0, \quad Q_{\lambda \lambda}(u, v) \leq 0,
\end{gathered}
$$

$7^{o} \bar{\eta} \leq \Gamma_{y}(u, v) \leq \eta,=\bar{\zeta} \leq \Delta_{y}(u, v) \leq \zeta, \quad \bar{L}+\bar{\zeta}-\zeta+\bar{\eta}-\eta \geq 0, \quad \bar{\vartheta} \leq \Phi_{\lambda}(t, \bar{u}, \bar{v}) \leq \vartheta$, $\bar{\mu} \leq \Psi_{\lambda}(t, \bar{u}, \bar{v}) \leq \mu, \quad \bar{M}+\bar{\vartheta}-\vartheta+\bar{\mu}-\mu \geq 0$ for $(u, v) \in \bar{\Omega}, \quad(t, \bar{u}, \bar{v}) \in \Omega$.

Then there exist monotone sequences $\left\{y_{n}\right\},\left\{z_{n}\right\},\left\{\lambda_{n}\right\},\left\{\gamma_{n}\right\}$ which converge uniformly to the unique solution of problem (3.1) and the convergence is quadratic.

Remark 3. Note that Theorem 4 contains, as a special case, the result of Theorem 3 if we put $\Phi(t, u, v)=\Psi(t, u, v)=0$ for $(t, u, v) \in \Omega$, and $\Delta(\bar{u}, \bar{v})=\Gamma(\bar{u}, \bar{v})=0$ for $(\bar{u}, \bar{v}) \in \bar{\Omega}$. Finally, we study the situation when problem (3.1) is replaced by the following one

$$
\left\{\begin{array}{c}
x^{\prime}(t)=\bar{f}(t, x(t), \lambda), t \in J, \quad x(0)=k_{0}, \\
0=\bar{G}(x(b), \lambda)
\end{array}\right.
$$

with $\bar{f}=f+\tilde{f}+g+\tilde{g},=\bar{G}=G+\tilde{G}+H+\tilde{H}$. We get weak quadratic convergence only if we assume that $f, g \in=C^{0,2,2}(\Omega, R), G, H \in C^{2}(\bar{\Omega}, R), \tilde{f}, \tilde{g} \in C^{0,1,1}(\Omega, R)$, = $\tilde{G}, \tilde{H} \in C^{1}(\bar{\Omega}, R)$. This general case is considered in the next theorem.

Theorem 5 (see [7]). Let $f, g \in C^{0,2,2}(\Omega, R), \quad G, H \in C^{2}(\bar{\Omega}, R), \quad \tilde{f}, \tilde{g} \in C(\Omega, R)$, = $\tilde{G}, \tilde{H} \in C(\bar{\Omega}, R)$. Assume that:

$1^{\circ}\left(y_{0}, \lambda_{0}\right),\left(z_{0}, \gamma_{0}\right) \in C^{1}(J, R) \times R$ are lower and upper solutions of problem (4.1), respectively, and such that $y_{0}(t) \leq z_{0}(t), t \in J$ and $\lambda_{0} \leq \gamma_{0}$,

$2^{\circ}$ conditions $5^{\circ}$ and $6^{\circ}$ of Theorem 4 hold,

$3^{o}-G_{\lambda}(u, v) \geq K_{1}, \quad-H_{\lambda}(u, v) \geq K_{2}, \bar{L}_{1} \leq G_{y}(u, v) \leq L_{1}, \quad \bar{L}_{2} \leq H_{y}(u, v) \leq L_{2}$, $\bar{M}_{1} \leq f_{\lambda}(t, \bar{u}, \bar{v}) \leq M_{1}, \quad \bar{M}_{2} \leq g_{\lambda}(t, \bar{u}, \bar{v}) \leq M_{2}$ for $(u, v) \in \bar{\Omega}, \quad(t, \bar{u}, \bar{v}) \in \Omega ;$ $\left|f_{y}(t, u, v)\right| \leq N_{1}, \quad\left|g_{y}(t, u, v)\right| \leq N_{2}$ for $(t, u, v) \in \Omega$,

$5^{o} \tilde{F}_{y}, \tilde{F}_{\lambda}, \tilde{\Phi}_{y},=\tilde{\Phi}_{\lambda}, \tilde{P}_{y}, \tilde{P}_{\lambda}, \tilde{\Delta}_{y}, \tilde{\Delta}_{\lambda}$ exist, are nondecreasing in the last two variables, while $\tilde{T}_{y}, \tilde{T}_{\lambda}, \tilde{\Psi}_{y}, \tilde{\Psi}_{\lambda}, \tilde{Q}_{y}, \tilde{Q}_{\lambda}, \tilde{\Gamma}_{y}, \tilde{\Gamma}_{\lambda}$ exist and are nonincreasing in the last two variables with $\tilde{F}=\tilde{f}+\tilde{\Phi}, \tilde{T}=\tilde{g}+\tilde{\Psi}, \tilde{P}=\tilde{G}+\tilde{\Delta},=\tilde{Q}=\tilde{H}+\tilde{\Gamma}$,

$6^{o} \bar{M}_{3} \leq \tilde{f}_{\lambda}(t, \bar{u}, \bar{v}) \leq M_{3}, \bar{M}_{4} \leq \tilde{g}_{\lambda}(t, \bar{u}, \bar{v}) \leq M_{4}$,

$K_{3} \leq-\tilde{G}_{\lambda}(u, v) \leq \bar{K}_{3}, K_{4} \leq-\tilde{H}_{\lambda}(u, v) \leq \bar{K}_{4}, \quad \bar{L}_{3} \leq \tilde{G}_{y}(u, v) \leq L_{3}, \quad \bar{L}_{4} \leq$ $\tilde{H}_{y}(u, v) \leq L_{4}, \quad\left|\tilde{f}_{y}(t, \bar{u}, \bar{v})\right| \leq N_{3},\left|\tilde{g}_{y}(t, \bar{u}, \bar{v})\right| \leq N_{4}, \bar{\eta}_{1} \leq \tilde{\Gamma}_{y}(u, v) \leq \eta_{1}, \bar{\zeta}_{1} \leq$ $\tilde{\Delta}_{y}(u, v) \leq \zeta_{1}, \bar{\vartheta}_{1} \leq \tilde{\Phi}_{\lambda}(t, \bar{u}, \bar{v}) \leq \vartheta_{1}, \bar{\mu}_{1} \leq \tilde{\Psi}_{\lambda}(t, \bar{u}, \bar{v}) \leq \mu_{1}$ for $(u, v) \in \Omega,(t, \bar{u}, \bar{v}) \in$ 
$7^{o}$ put $L=L_{1}+L_{2}+L_{3}+L_{4}, K=K_{1}+K_{2}+K_{3}+K_{4}>0, M=M_{1}+M_{2}+M_{3}+M_{4}$, $N=N_{1}+N_{2}+N_{3}+N_{4}, \bar{L}=\bar{L}_{1}+\bar{L}_{2}+\bar{L}_{3}+\bar{L}_{4}+\bar{\zeta}_{1}-\zeta_{1}+\bar{\eta}_{1}-\eta_{1}, \quad \bar{M}=$ $\bar{M}_{1}+\bar{M}_{2}+\bar{M}_{3}+\bar{M}_{4}+\bar{\vartheta}_{1}-\vartheta_{1}+\bar{\mu}_{1}-\mu_{1}$,

$8^{\circ}$ condition $7^{\circ}$ of Theorem 4 holds,

$9^{\circ} \quad S(b)<1$, where $S$ is defined as in condition $4^{\circ}$ of Theorem 3 .

Then there exist monotone sequences $\left\{y_{n}\right\},\left\{z_{n}\right\},\left\{\lambda_{n}\right\},\left\{\gamma_{n}\right\}$ which converge uniformly and monotonically to the unique solution of problem (4.1) and the convergence is semi-quadratic, i.e.,

$$
\begin{gathered}
\max _{t \in J}\left|x(t)-y_{n+1}(t)\right| \leq a_{1} \max _{t \in J}\left|x(t)-y_{n}(t)\right|^{2}+a_{2} \max _{t \in J}\left|x(t)-z_{n}(t)\right|^{2}+a_{3}\left|\lambda-\lambda_{n}\right|^{2} \\
+a_{4}\left|\lambda-\gamma_{n}\right|^{2}+a_{5} \max _{t \in J}\left|x(t)-y_{n}(t)\right|+a_{6}\left|\lambda-\lambda_{n}\right|, \\
\left|\lambda-\lambda_{n+1}\right| \leq b_{1} \max _{t \in J}\left|x(t)-y_{n}(t)\right|^{2}+b_{2} \max _{t \in J}\left|x(t)-z_{n}(t)\right|^{2}+b_{3}\left|\lambda-\lambda_{n}\right|^{2} \\
+b_{4}\left|\lambda-\gamma_{n}\right|^{2}+b_{5} \max _{t \in J}\left|x(t)-y_{n}(t)\right|+b_{6}\left|\lambda-\lambda_{n}\right|, \\
\max _{t \in J}\left|x(t)-z_{n+1}(t)\right| \leq \bar{a}_{1} \max _{t \in J}\left|x(t)-y_{n}(t)\right|^{2}+\bar{a}_{2} \max _{t \in J}\left|x(t)-z_{n}(t)\right|^{2}+\bar{a}_{3}\left|\lambda-\lambda_{n}\right|^{2} \\
+\bar{a}_{4}\left|\lambda-\gamma_{n}\right|^{2}+\bar{a}_{5} \max _{t \in J}\left|x(t)-z_{n}(t)\right|+\bar{a}_{6}\left|\lambda-\gamma_{n}\right| \\
\left|\lambda-\gamma_{n+1}\right| \leq \bar{b}_{1} \max _{t \in J}\left|x(t)-y_{n}(t)\right|^{2}+\bar{b}_{2} \max _{t \in J}\left|x(t)-z_{n}(t)\right|^{2}+\bar{b}_{3}\left|\lambda-\lambda_{n}\right|^{2} \\
+\bar{b}_{4}\left|\lambda-\gamma_{n}\right|^{2}+\bar{b}_{5} \max _{t \in J}\left|x(t)-z_{n}(t)\right|+\bar{b}_{6}\left|\lambda-\gamma_{n}\right|,
\end{gathered}
$$

for some nonnegative constants $a_{i}, b_{i}, \bar{a}_{i}, \bar{b}_{i}, i=1,2,3,4,5,6$.

\section{REFERENCES}

[1] Goma, A.: The method of successive approximations in a two-point boundary value problem with a parameter, Ukr. Mat. Zh., 29, (1977), 800-807. (in Russian)

[2] JANkowski, T.: Existence, uniqueness and approximate solutions of problems with a parameter, Zesz. Nauk. Politech. Gdansk., Mat., 16, (1993), 3-167.

[3] Jankowski, T. and Lakshmikantham, V.: Monotone iterations for differential equations with a parameter, J. Appl. Math. Stoch. Anal., 10, (1997), 273-278.

[4] Jankowski, T.: Monotone iterations for first order differential equations with a parameter, Acta Math. Hungar., 84, (1999), 65-80.

[5] Jankowski, T. and McRAe, F. A.: An extension of the method of quasilinearization for differential problems with a parameter, Nonlinear Studies, 6, (1999), 21-44.

[6] Jankowski, T.: Generalizations of the method of quasilinearization for differential problems with a parameter, Dynamic Systems and Applications, 8, (1999), 53-72.

[7] Jankowski, T.: Generalization of the method of quasilinearization, International Journal of Non-linear Differential Equations, 5, (1999), 122-147. 
[8] Kurpiel, N. S. And Marusiak, A. G.: A multipoint boundary value problem for differential equations with parameters, Ukr. Mat. Zh., 32, (1980), 223-226. (in Russian)

[9] Ladde, G. S., Lakshmikantham, V. and Vatsala, A. S.: Monotone Iterative Techniques for Nonlinear Differential Equations, Pitman, 1985.

[10] Lakshmikantham, V. and Vatsala, A. S.: Generalized Quasilinearization for Nonlinear Problems, Kluwer, Dordrecht, 1998.

[11] Lakshmikantham, V. and Malek, S.: Generalized quasilinearization, Nonlinear World, 1, (1994), 59-63.

[12] Lakshmikantham, V., Leela, S. and Sivasundaram, S.: Extensions of the method of quasilinearization, J. Otimization Theory Appl., 87, (1995), 379-401.

[13] Lakshmikantham, V., Leela, S. and McRae, F.A.: Improved generalized quasilinearization method, Nonlinear Analysis 24, (1995), 1627-1637.

[14] Lakshmikantham, V. and Shahzad, N.: Further generalization of generalized quasilinearization method, J. Appl. Math. Stoch. Anal., 7, (1994), 545-552.

[15] Pomentale, T.: A constructive theorem of existence and uniqueness for the problem $y^{\prime}=f(x, y, \lambda), y(a)=\alpha, y(b)=\beta$, Z. Angew. Math. Mech., 56, (1976), 387-388.

[16] ZaWischa, K.: Über die Differentialgleichung $y^{\prime}=k f(x, y)$ deren Lösungskurve durch zwei gegebene Punkte hindurchgehen soll, Monatsh. Math. Phys., 37, (1930), 103-124. 